\title{
Efficacy and Safety of Argon Plasma Coagulation for Hemorrhagic Chronic Radiation Proctopathy: A Systematic Review
}

\author{
Yanan Peng, 1,2 Haizhou Wang, ${ }^{1,2}$ Juerong Feng, ${ }^{1,2}$ Shilin Fang, ${ }^{1,2}$ Meng Zhang, ${ }^{1,2}$ \\ Fan Wang, ${ }^{1,2}$ Ying Chang, ${ }^{1,2}$ Xianyan Shi, ${ }^{1,2}$ Qiu Zhao, ${ }^{1,2}$ and Jing Liu $\mathbb{D}^{1,2}$ \\ ${ }^{1}$ Department of Gastroenterology, Zhongnan Hospital of Wuhan University, Wuhan, China \\ ${ }^{2} \mathrm{Hubei}$ Clinical Center and Key Lab of Intestinal and Colorectal Diseases, Wuhan, China \\ Correspondence should be addressed to Jing Liu; liujing81@hotmail.com
}

Received 20 July 2017; Revised 13 November 2017; Accepted 8 January 2018; Published 25 February 2018

Academic Editor: Joanne Bowen

Copyright (c) 2018 Yanan Peng et al. This is an open access article distributed under the Creative Commons Attribution License, which permits unrestricted use, distribution, and reproduction in any medium, provided the original work is properly cited.

\begin{abstract}
Hemorrhagic chronic radiation proctopathy (CRP) is a common complication after pelvic radiotherapy in patients with prostate or gynecological cancers. This systematic review was conducted to evaluate the efficacy and safety of argon plasma coagulation (APC) in treating hemorrhagic CRP. The databases of PubMed, Embase, and Cochrane Library were searched for related studies from inception to July 2017. Finally, 33 studies were identified with a total of 821 hemorrhagic CRP patients. After APC treatment, hemoglobin levels increased from $7.7-13.4 \mathrm{~g} / \mathrm{L}$ to $11-14 \mathrm{~g} / \mathrm{L}$ (including 15 studies). All $(n=33)$ studies reported an effective rate in rectal bleeding, among which five studies had a rate of $100 \%$. Short-term complications were reported in 31 studies, while long-term complications in 33 studies and no complication in 11 studies. As for the severe complications, perforation was reported by 2 out of 33 studies, and the incidences were $3.3 \%(1 / 30)$ and $3.7 \%(1 / 27)$, respectively. As for APC setting, argon gas flow rate (median $1.5 \mathrm{~L} / \mathrm{min}$ ) and electric power (median $50 \mathrm{~W}$ ) had no significant influence on complications and hemostasis. In conclusion, current literature indicated that APC therapy was an effective and safe strategy for hemorrhagic CRP, and large-scale prospective studies are needed to warrant our study.
\end{abstract}

\section{Introduction}

Hemorrhagic chronic radiation proctopathy $(\mathrm{CRP})$ is a common complication after pelvic radiotherapy. It had an incidence of $5 \%$ to $15 \%$ in the patients of pelvic cancers within 6 months after radiotherapy [1]. Multiple factors were involved in the pathogenesis of hemorrhagic CRP, like mucosal damage, microvascular injury with tissue ischemia, and telangiectasias [2]. Oral or enema treatment with salicylates, corticosteroids, and sucralfate usually had a limited benefit. Moreover, surgery was not recommended for a high incidence of morbidity [3]. However, hyperbaric oxygen therapy (HOT) and formalin application were reported to be effective in radiation proctitis [3-8]. As an easy method in destructing the telangiectasias caused by radiotherapy, argon plasma coagulation (APC) was reported to have a better efficacy than formalin in treating hemorrhagic CRP (79\% versus $27 \%, P=0.017$ ) [4]. Alvaro et al. also found that APC therapy had a significantly better response than hyperbaric oxygen therapy in reducing blood transfusion and tissue toxicity, although both treatments showed no significant difference in resolving rectal bleeding. In spite of this, several studies still reported a high incidence of up to $20 \%$ in APCassociated complications, such as ulcers, perforations, strictures, and fistulas [5-10]. Furthermore, Sato et al. found that appropriate APC settings (e.g., electric power, application time, and argon flow rate) could reduce the damage to deeper tissues, thus decreasing the incidence of complications [11]. However, its effectiveness and safety were not clearly assessed in previous studies. Therefore, we conducted a systematic review to evaluate the efficacy and safety of APC in treating hemorrhagic CRP. 


\section{Methods}

2.1. Medical Literature Search. The databases of PubMed, Embase, and Cochrane Library were searched for relevant studies from inception to July 2017, using the key words including "radiation proctopathy" [MeSH Terms] OR ("radiation" [All Fields] AND "proctopathy" [All Fields]) OR "radiation proctopathy" [All Fields] OR ("radiation proctitis" [MeSH Terms]) OR ("radiation" [All Fields] AND "proctitis"[All Fields]) OR ("radiation proctitis" [All Fields]) AND ("Argon plasma coagulation therapy" [MeSH Terms] OR ("Argon plasma coagulation" [All Fields] AND "therapy" [All Fields]) OR "Argon plasma coagulation therapy" [All Fields]) OR ("APC therapy”[MeSH Terms]) OR ("APC therapy" [All Fields]). There was no language restriction for literature search.

2.2. Selection Criteria and Exclusion Criteria. All the studies were reviewed independently by two investigators. Studies were included if fulfilling the following criteria: (i) including patients with pelvic cancers and subsequent radiotherapy, (ii) diagnosed as hemorrhagic CRP, and (iii) evaluated the efficacy and safety of APC. The exclusion criteria were as follows: reviews, case reports, animal studies, and studies without full text or sufficient data.

2.3. Data Extraction and Quality Assessment. The following information was extracted from each included study: author, year of publication, area, study design, number of cases, age, gender, cancer type, follow-up time, argon gas flow rate and electric power of APC, number of rectal bleeding cessation (NRBC), hemoglobin (LHb) levels, and short-term and long-term complications. The quality of included studies were assessed using an adjusted version of the NewcastleOttawa Scale, which contained a cohort selection on APC administration and its representativeness, ascertainment of APC exposure, and evidence that there was no prior exposure to APC, outcome, longevity follow-up (at least 6 weeks), and bias due to dropout or incomplete follow-up [12].

2.4. Statistical Analysis. The efficacy of APC therapy was evaluated according to the degree of improvement in rectal bleeding and hemoglobin, while the safety was assessed based on the incidence of complications, which were categorized as short term (e.g., urgency, diarrhea, incontinence, fever, anal or abdominal pain, and perforation) and long term (e.g., stenosis/strictures, rectal ulcers, recurrence of rectal bleeding, and fistula). As for APC settings, electric power, argon gas flow, and coagulation time were included analysis. Studies with a score of $\geq 5$ were considered as high quality, with 3-4 for moderate quality. Discrepancies of assessments were resolved through discussion.

\section{Results}

3.1. Study Characteristics. Of the 167 papers identified at the initial search, after removing the duplicates and exclusion of irrelevant studies, comments, case reports, and reviews, 37 studies were selected for the review of the full text. Another 4 studies were excluded due to the absence of certain interested outcomes including rectal bleeding cessation, the improvement of hemoglobin, and short-term and longterm complications, and 33 studies were included in the final review (Figure 1). All included studies including prospective $(n=21)$ and retrospective $(n=8)$ uncontrolled cohort trials, and 4 nonrandomized control trials [4, 13-15], were conducted at a single academic center. The average time of follow-up was 28 months (1 month to 170 months) (Table 1). All patients with chronic radiation proctitis were characterized by rectal bleeding, which was referred to as hemorrhagic CRP.

3.2. Efficacy of $A P C$. The improvements in rectal bleeding and hemoglobin were regarded as efficacy of APC treatment, which were observed in 821 and 383 patients, respectively. 15 studies reported the improvement of hemoglobin after APC administration. Table 2 demonstrates that after APC treatment, the mean of hemoglobin levels was improved from $7.7-13.4 \mathrm{~g} / \mathrm{L}$ to $11-14 \mathrm{~g} / \mathrm{L} .33$ studies documented rectal bleeding cessation as evidenced in Table 2, 5 of which had a $100 \%$ hemostasis rate $[5,7-9,16]$. Furthermore, in the largest study, Sato et al. [11] reported that during a mean follow-up of 34.6 months, 4 patients $(6.3 \%)$ had minor recurrent rectal bleeding and $60(93.8 \%)$ remained in remission. $(P=0.002)$ (Table 2).

3.3. Safety of APC. APC-associated short-term and long-term complications were shown in Table 3. Short-term complications were reported in 31 studies, while long-term complications in 33 studies and no complication in 11 studies. The incidence of complications was $0-63.6 \%$. Especially in a study from Venkatesh and Ramanujam, USA, there was no complication among 40 patients after APC therapy [17], because the team had experience. The commonest procedure-related short-term complications reported were anal or rectal pain with or without tenesmus, which was most likely to occur following treatment near the dentate line. Abdominal bloating, vomiting, adynamic ileus, vagal symptoms, cramping, incontinence, fever, and colonic explosion were also reported. Necrosis of the lower part of the rectum was reported in one study [10]. The severe complication of perforation was reported in 2 series $[10,18]$, the incidence of which was $3.7 \%(1 / 27)$ and $3.3 \%(1 / 30)$, respectively. Ben-Soussan et al. reported that one patient had, during APC, colonic explosion which immediately led to a perforation [18]. The patient needed surgical treatment and made a complete recovery in 2 weeks. At the same time, Ben mentioned that the incidence of bowel explosion was higher after local preparation in comparison with oral reparation $(P<0.05)$. Enema preparation with persistent solid stool above the coagulated lesions contributed to the main risk of perforation. Canard et al. also reported 1 perforation [10]. But we could not obtain the detailed information about the treatment of perforation and prognosis of the patient from their study. Grund et al. reported 1600 applications of APC for a variety of indications in the upper and lower gastrointestinal tract and reported a perforation rate of $0.31 \%$ [19]. 


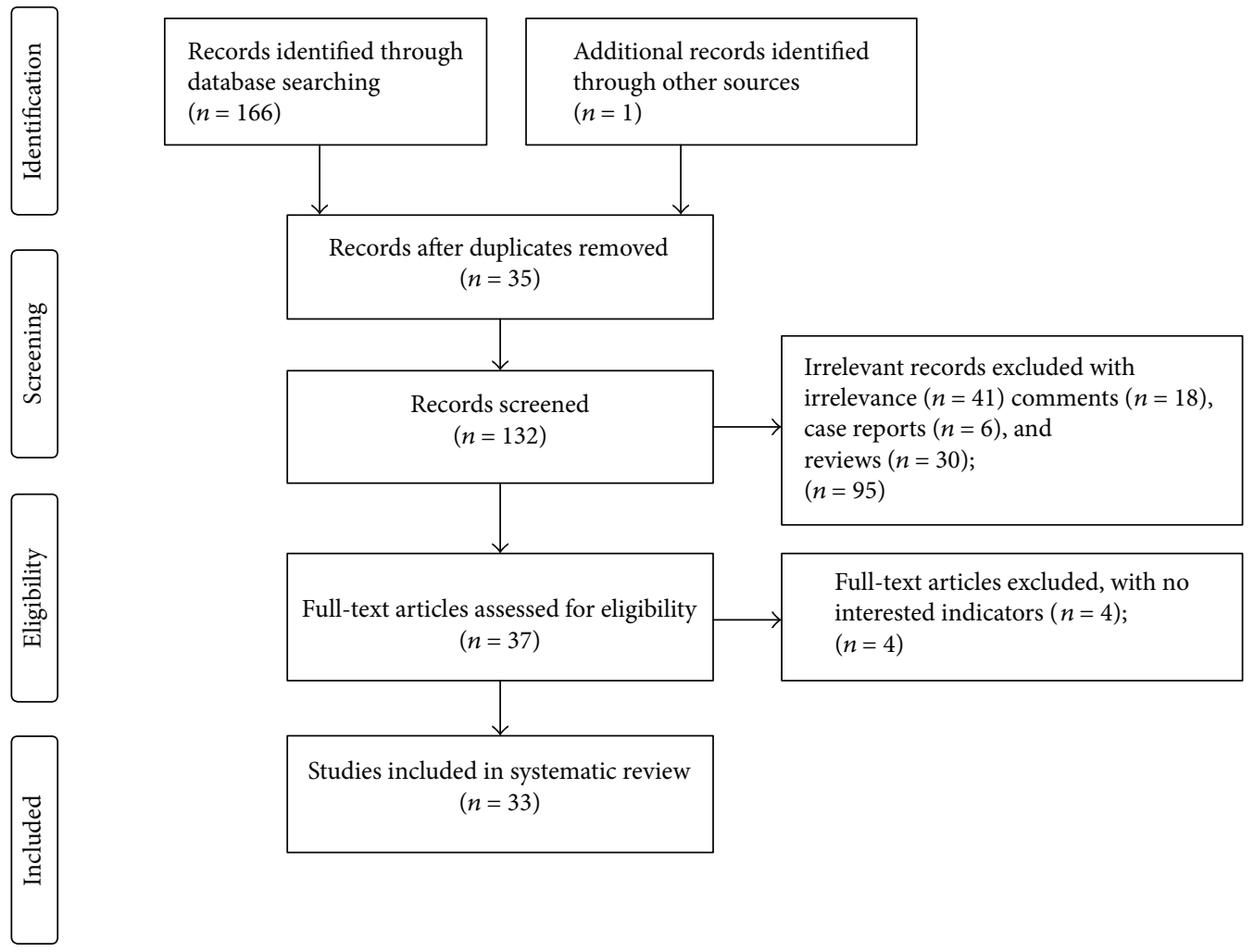

FIGURE 1: Flow diagram of the identification of the studies for inclusion in the systematic review.

Rectal ulcers, stenosis, and recurrence of rectal bleeding were common long-term complications following APC treatment. Chruscielewska-Kiliszek et al. reported rectal ulcers in $35(56.5 \%)$ of 62 patients [20], an incidence that was relatively high in comparison with the reported overall rate of about 3.3\% (1/30) [14]-21.4\% [13] (Table 3) in other series. Rectal ulcers developing during APC could be considered a consequence of thermal injury to already damaged and vascularly compromised tissue that was thus more fragile and had poorer healing [21]. The fact that rectal ulcers were not clinically troublesome meant they should not be considered an absolute contraindication to APC nor do they necessarily require any additional endoscopic follow-up [22]. The occurrence of stenosis compared with rectal ulcer was less common. The incidence of rectal stenosis varied among different studies, many studies describing no occurrence of rectal stricture while few studies reporting such complication in $2 \%(1 / 49)[23]-13.3 \%(2 / 15)$ [7]. However, given the fact that most of the rectal strictures were asymptomatic, their true incidence was difficult to estimate and theoretically would be higher than reported by several studies. The recurrence rate of rectal bleeding ranged from $2.1 \%(1 / 48)$ [24] to $6.3 \%(1 / 16)$ [6]. Patients on anticoagulants or aspirin were more likely to relapse. Kaassis et al. reported that patients who were receiving anticoagulation therapy required more APC sessions but could also achieve an equivalent therapeutic efficacy as those who were not on anticoagulation [6].

3.4. Arguments of APC Setting. By reviewing relative literatures, we realized that different studies had different optional
APC settings. This systematic review showed arguments of APC settings in 32 trials (Table 1). The electric power setting ranged from 25 to $80 \mathrm{~W}$ (median $50 \mathrm{~W}$ ), with an argon flow rate from 0.6 to $3.0 \mathrm{~L} / \mathrm{min}$ (median $1.5 \mathrm{~L} / \mathrm{min}$ ). Of 10 trials that reported the records of coagulation time, only one trial briefly provided the specific number of coagulation time. The range of coagulation time was from $0.5 \mathrm{~s}$ to $3 \mathrm{~s}$. Among included studies that observed incidence of complications, regardless of the range of electric power setting (30-50 W versus $50-80 \mathrm{~W})$, the corresponding rate of complications had no difference $(0-58.1 \%$ versus $0-63.6 \%)$. Even with the same power $(60 \mathrm{~W})$ and the flow rate $>1.5 \mathrm{~L} / \mathrm{min}, 4$ series, respectively, reported complications in $0 \%, 35.7 \%, 0 \%$, and $13.3 \%[5,7,13,25]$. In addition, there were no uniform settings of APC in 5 series which reported 100\% hemostasis rate. Therefore, APC settings appeared to be uncorrelated with the incidence of complications in our study.

3.5. Quality Assessment. The quality assessment of studies using NOS is shown in Table 4. The qualities of studies were considered high for 31 studies and moderate for 2 studies.

\section{Discussion}

Chronic radiation proctopathy had an incidence of 5\%-15\% in patients with pelvic radiotherapy, and rectal bleeding was the most common complication. Radiation injury to the rectal wall became apparent like obliterative endarteritis with secondary tissue ischemic and development of neovascular mucosal lesions. These ones could bleed in a delayed fashion 


\begin{tabular}{|c|c|c|c|c|c|c|c|c|c|c|c|c|c|c|}
\hline 产 & 0 & 0 & 0 & 0 & a & 0 & 0 & a & 0 & 0 & 0 & 0 & 0 & 。 \\
\hline 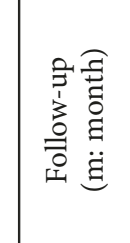 & 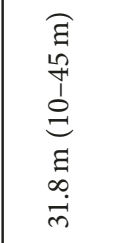 & $\Xi$ & ઘ & 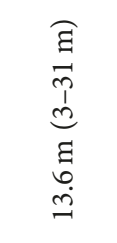 & 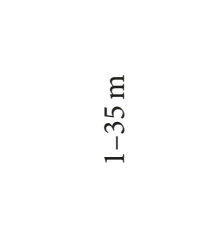 & 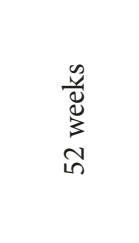 & 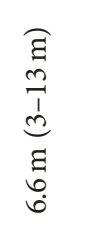 & $\dddot{\Xi}$ & 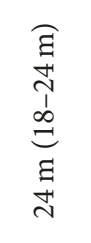 & 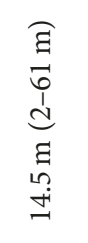 & 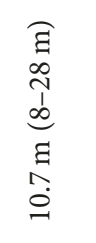 & 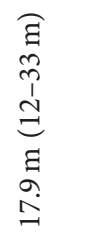 & $\begin{array}{l}\Xi \\
\sigma \\
\omega \\
+1 \\
1 \\
\infty \\
\infty \\
\sim\end{array}$ & 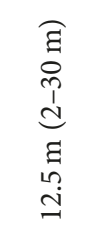 \\
\hline 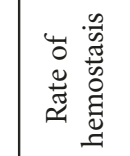 & 定 & $\ddot{\infty}$ & $\begin{array}{l}\text { L } \\
\text { S }\end{array}$ & ลे & ณू & $\hat{\sigma}$ & $\stackrel{\widehat{\Omega}}{\widehat{\alpha}}$ & 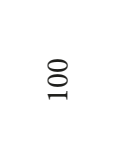 & 8 & $\stackrel{\circ}{\circ}$ & 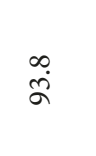 & $\stackrel{m}{\infty}$ & $\begin{array}{l}\infty \\
\dot{\infty} \\
\infty\end{array}$ & ๙a. \\
\hline 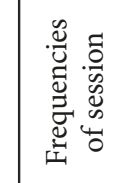 & 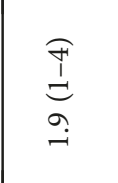 & $\stackrel{\infty}{\stackrel{\infty}{\rightarrow}}$ & $\overrightarrow{+1}$ & $\begin{array}{l}\underset{I}{=} \\
\stackrel{0}{0} \\
\stackrel{0}{i}\end{array}$ & $\begin{array}{l}\widehat{D} \\
\stackrel{1}{D} \\
\stackrel{n}{n} \\
i \\
o \\
0\end{array}$ & $\underset{\sim}{\stackrel{T}{P}}$ & $\stackrel{\widehat{I}}{\stackrel{-}{=}}$ & 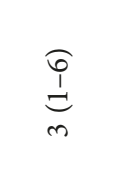 & $\begin{array}{l}\stackrel{f}{d} \\
\underset{\sim}{d}\end{array}$ & $n$ & $\stackrel{r}{\dot{m}}$ & $\begin{array}{l}\underset{\infty}{\stackrel{p}{N}} \\
\stackrel{\sim}{N}\end{array}$ & $\begin{array}{l}\hat{i} \\
+1 \\
+1 \\
0 \\
\dot{m}\end{array}$ & 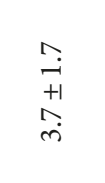 \\
\hline 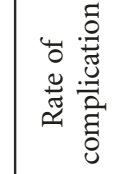 & : & $\begin{array}{l}\stackrel{\circ}{\circ} \\
\stackrel{\text { İ }}{+}\end{array}$ & $\begin{array}{l}\stackrel{\circ}{\circ} \\
\hat{\omega} \\
\ddot{n}\end{array}$ & 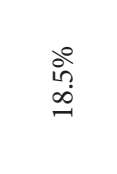 & 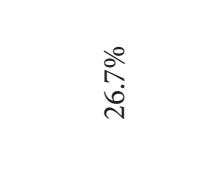 & $\begin{array}{l}\stackrel{\circ}{\infty} \\
\text { in } \\
\text { in }\end{array}$ & $\stackrel{\circ}{0}$ & $\stackrel{\text { ò }}{\text { ì }}$ & ذें & 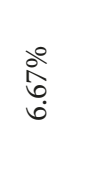 & $\underset{\stackrel{\circ}{m}}{\stackrel{m}{m}}$ & なें & ذँ. & ஓे \\
\hline 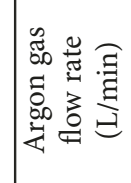 & 先 & $\stackrel{\stackrel{I}{I}}{I}$ & $\stackrel{\circ}{-}$ & $\begin{array}{c}\overrightarrow{1} \\
\infty \\
0\end{array}$ & 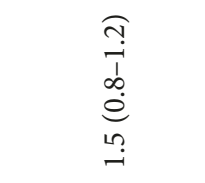 & 年 & $\dddot{g}$ & $\sim$ & m & $\stackrel{\infty}{\rightarrow}$ & $\stackrel{0}{0}$ & $N$ & $\stackrel{\infty}{\stackrel{\infty}{+}}$ & - \\
\hline 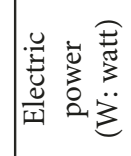 & 8 & $\begin{array}{l}0 \\
b \\
b \\
q\end{array}$ & 8 & $\begin{array}{l}0 \\
\text { h } \\
\text { an }\end{array}$ & 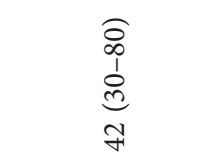 & 勇 & $\cong$ & in & 8 & in & 아 & 아 & $\begin{array}{l}8 \\
1 \\
1 \\
0\end{array}$ & 아 \\
\hline 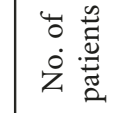 & 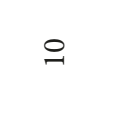 & \pm & \pm & ते & m & $\widetilde{\sigma}$ & $\simeq$ & in & $n$ & in & $\stackrel{\bullet}{\sim}$ & i & $\stackrel{\infty}{\infty}$ & \\
\hline 芯 & 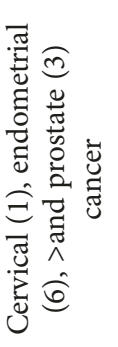 & $\dddot{g}$ & $\Xi$ & 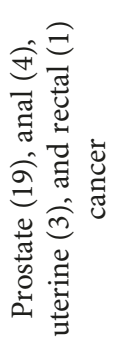 & 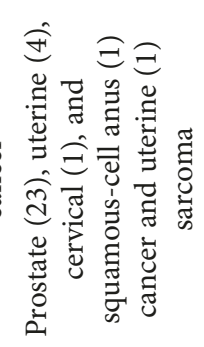 & 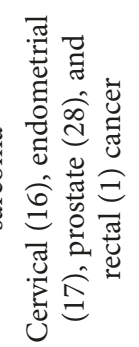 & 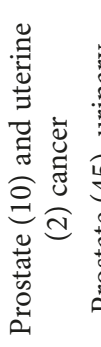 & 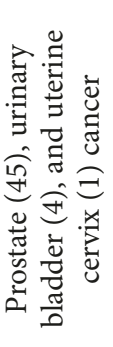 & 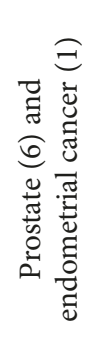 & 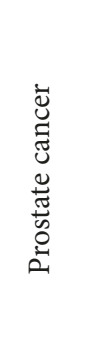 & 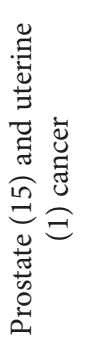 & 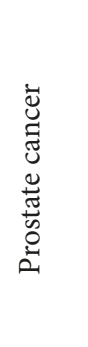 & 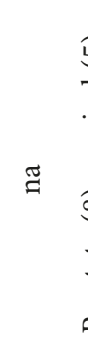 & 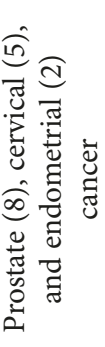 \\
\hline 总 & $\begin{array}{l}0 \\
0 \\
1 \\
0 \\
0 \\
\infty \\
0 \\
0\end{array}$ & 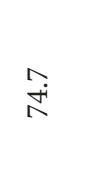 & 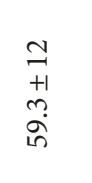 & $\begin{array}{l}0 \\
\infty \\
0 \\
1 \\
0 \\
0 \\
-1 \\
\infty\end{array}$ & $\begin{array}{l}\widehat{0} \\
0 \\
0 \\
0 \\
0 \\
\\
\hat{R} \\
\hat{R}\end{array}$ & $\begin{array}{l}0 \\
\stackrel{0}{0} \\
+1 \\
+1 \\
02 \\
0 \\
0\end{array}$ & $\begin{array}{l}\widehat{D} \\
\infty \\
+0 \\
\mathbb{d} \\
\mathbb{N}\end{array}$ & 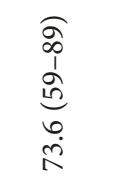 & $\stackrel{\Xi}{7}$ & $\begin{array}{l}\widehat{\infty} \\
\hat{1} \\
b \\
0 \\
\stackrel{0}{R} \\
R\end{array}$ & $\begin{array}{l}\widehat{O} \\
1 \\
1 \\
\tilde{d} \\
1 \\
\tilde{n} \\
\tilde{N}\end{array}$ & 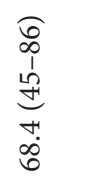 & 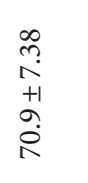 & 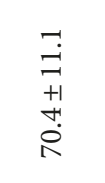 \\
\hline 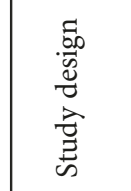 & 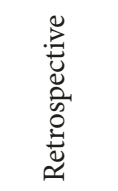 & 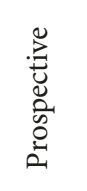 & 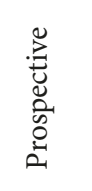 & 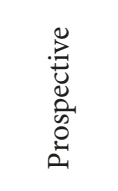 & 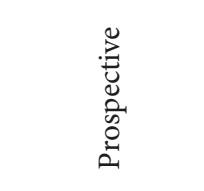 & 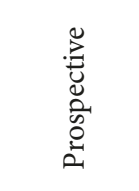 & 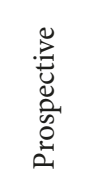 & 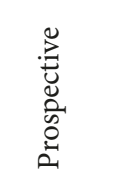 & 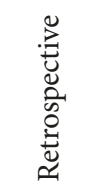 & 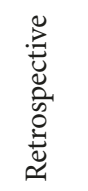 & 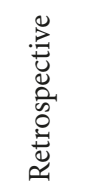 & 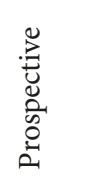 & 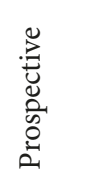 & 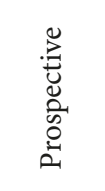 \\
\hline 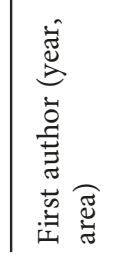 & 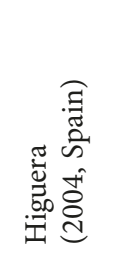 & 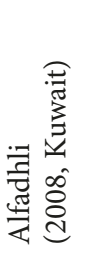 & 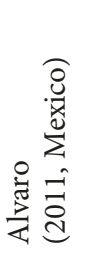 & 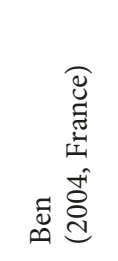 & 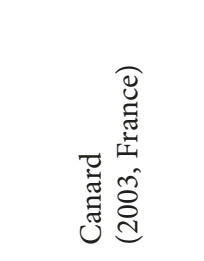 & 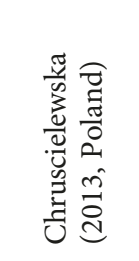 & 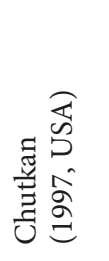 & 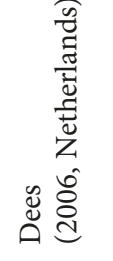 & 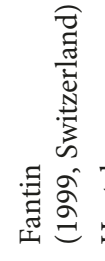 & 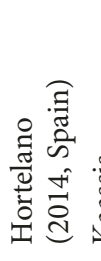 & 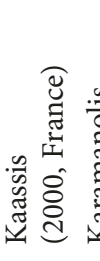 & 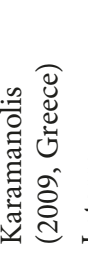 & 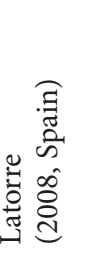 & 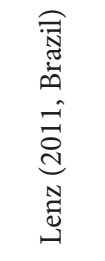 \\
\hline
\end{tabular}




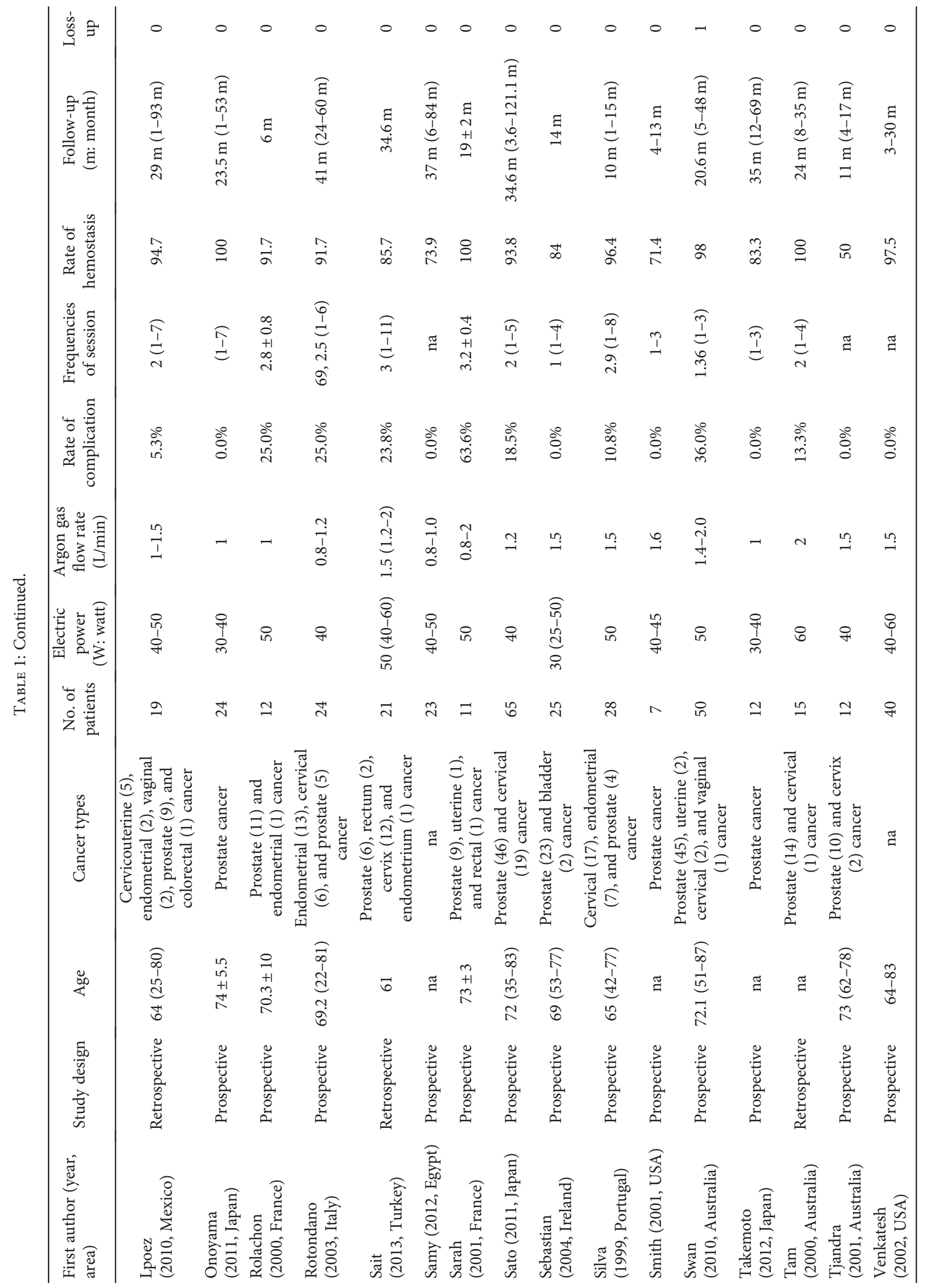




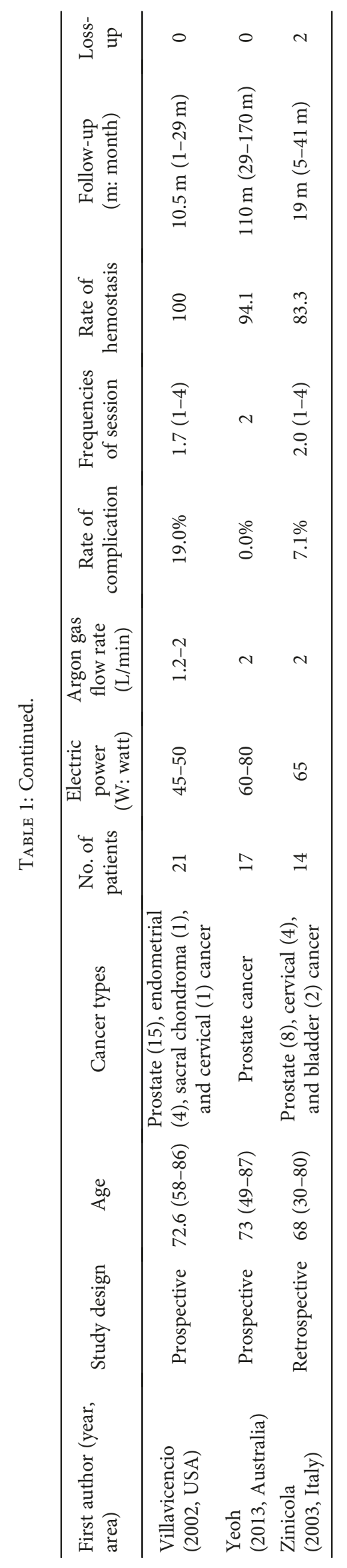


TABLE 2: Study characteristics of selected studies on the levels of hemoglobin and rectal bleeding before and after APC administration.

\begin{tabular}{|c|c|c|c|c|c|c|c|c|}
\hline Author & Year & Type of study & Proctitis & $\begin{array}{c}\text { Total } \\
\text { number of } \\
\text { bleeding }\end{array}$ & $\begin{array}{c}\text { Number } \\
\text { of } \\
\text { rebleeding }\end{array}$ & $\begin{array}{c}\text { Rate of } \\
\text { hemostasis } \\
(\%)\end{array}$ & $\begin{array}{c}\text { Mean level of } \\
\text { hemoglobin before } \\
\text { APC }(\mathrm{g} / \mathrm{dL}) \pm \mathrm{SD}\end{array}$ & $\begin{array}{c}\text { Mean level of } \\
\text { hemoglobin after } \\
\text { APC }(\mathrm{g} / \mathrm{dL}) \pm \mathrm{SD}\end{array}$ \\
\hline Alfadhli & 2008 & Prospective & Prospective & 14 & 3 & 78.6 & na & na \\
\hline $\begin{array}{l}\text { Álvaro- } \\
\text { Villegas }\end{array}$ & 2011 & Prospective & $\begin{array}{l}\text { Chronic } \\
\text { radiation } \\
\text { proctitis }\end{array}$ & 14 & 2 & 85.7 & $9.9 \pm 2.3$ & $11.3 \pm 2$ \\
\hline Ben & 2004 & Prospective & $\begin{array}{l}\text { Hemorrhagic } \\
\text { radiation } \\
\text { proctitis }\end{array}$ & 27 & 2 & 92.6 & na & na \\
\hline Canard & 2003 & Prospective & $\begin{array}{l}\text { Radiation } \\
\text { proctitis }\end{array}$ & 28 & 2 & 92.9 & na & na \\
\hline Chruscielewska & 2013 & Prospective & $\begin{array}{l}\text { Chronic } \\
\text { radiation } \\
\text { proctitis }\end{array}$ & 62 & 3 & 95.2 & $13.07 \pm 1.73$ & $13.96 \pm 1.44$ \\
\hline Chutkan & 1997 & Prospective & Proctitis & 12 & 1 & 91.7 & na & na \\
\hline Dees & 2006 & Prospective & $\begin{array}{l}\text { Chronic } \\
\text { radiation } \\
\text { proctitis }\end{array}$ & 48 & 1 & 97.9 & na & na \\
\hline Fantin & 1999 & Retrospective & Proctitis & 7 & 0 & 100.0 & na & na \\
\hline $\begin{array}{l}\text { de la Serna } \\
\text { Higuera }\end{array}$ & 2004 & Retrospective & $\begin{array}{l}\text { Hemorrhagic } \\
\text { radiation } \\
\text { proctopathy }\end{array}$ & 10 & 1 & 90.0 & na & na \\
\hline Hortelano & 2013 & Prospective & $\begin{array}{l}\text { Chronic } \\
\text { radiation } \\
\text { proctitis }\end{array}$ & 30 & 7 & 76.7 & $9.6(5.1-14.1)$ & $11.65(10.2-14.6)$ \\
\hline Kaassis & 2000 & Retrospective & Proctitis & 16 & 1 & 93.8 & na & na \\
\hline Karamanolis & 2009 & Prospective & $\begin{array}{l}\text { Radiation } \\
\text { proctitis }\end{array}$ & 56 & 6 & 89.3 & na & na \\
\hline Latorre & 2008 & Prospective & $\begin{array}{l}\text { Chronic } \\
\text { radiation } \\
\text { proctopathy }\end{array}$ & 38 & 5 & 86.8 & $11.3 \pm 3.05$ & $14.014 \pm 1.29$ \\
\hline Lenz & 2011 & Prospective & $\begin{array}{c}\text { Chronic } \\
\text { radiation } \\
\text { coloproctopathy }\end{array}$ & 15 & 1 & 93.3 & $11.7 \pm 2.7$ & $13.0 \pm 0.9$ \\
\hline Lpoez & 2010 & Retrospective & $\begin{array}{l}\text { Radiation } \\
\text { proctopathy }\end{array}$ & 19 & 1 & 94.7 & $11.8(7.3-16.5)$ & $12.9(7.5-16.5)$ \\
\hline Onoyama & 2011 & Prospective & $\begin{array}{l}\text { Chronic } \\
\text { hemorrhagic } \\
\text { radiation } \\
\text { proctitis }\end{array}$ & 24 & 0 & 100.0 & $10 \pm 2.2$ & $12.3 \pm 1.5$ \\
\hline Rolachon A & 2000 & Prospective & $\begin{array}{l}\text { Proctitis and } \\
\text { proctosigmoiditis }\end{array}$ & 12 & 1 & 91.7 & $7.9 \pm 2.1$ & $11 \pm 1.4$ \\
\hline Rotondano & 2003 & Prospective & $\begin{array}{l}\text { Chronic } \\
\text { radiation } \\
\text { proctopathy }\end{array}$ & 24 & 2 & 91.7 & $9.2 \pm 2.4$ & $13.6 \pm 1.1$ \\
\hline Sait Dag & 2013 & Retrospective & $\begin{array}{l}\text { Radiation } \\
\text { proctitis }\end{array}$ & 21 & 3 & 85.7 & na & na \\
\hline Samy & 2012 & Prospective & Chronic proctitis & 23 & 6 & 73.9 & na & na \\
\hline Sarah & 2001 & Prospective & Proctitis & 11 & 0 & 100.0 & $7.7 \pm 2.8$ & $11.5 \pm 2.6$ \\
\hline Sato & 2011 & Prospective & $\begin{array}{l}\text { Hemorrhagic } \\
\text { radiation } \\
\text { proctopathy }\end{array}$ & 65 & 4 & 93.8 & $11.1(5.8-13.3)$ & $13.7(12-15.2)$ \\
\hline Sebastian & 2004 & Prospective & $\begin{array}{l}\text { Radiation } \\
\text { proctopathy }\end{array}$ & 25 & 4 & 84.0 & $10.05 \pm 2.21$ & $12.44 \pm 1.09$ \\
\hline Silva & 1999 & Prospective & Proctosigmoiditis & 28 & 1 & 96.4 & na & na \\
\hline
\end{tabular}


TABLE 2: Continued.

\begin{tabular}{|c|c|c|c|c|c|c|c|c|}
\hline Author & Year & Type of study & Proctitis & $\begin{array}{c}\text { Total } \\
\text { number of } \\
\text { bleeding }\end{array}$ & $\begin{array}{c}\text { Number } \\
\text { of } \\
\text { rebleeding }\end{array}$ & $\begin{array}{c}\text { Rate of } \\
\text { hemostasis } \\
(\%)\end{array}$ & $\begin{array}{c}\text { Mean level of } \\
\text { hemoglobin before } \\
\text { APC }(\mathrm{g} / \mathrm{dL}) \pm \mathrm{SD}\end{array}$ & $\begin{array}{l}\text { Mean level of } \\
\text { hemoglobin after } \\
\text { APC }(\mathrm{g} / \mathrm{dL}) \pm \mathrm{SD}\end{array}$ \\
\hline Smith & 2001 & Prospective & Proctitis & 7 & 2 & 71.4 & na & na \\
\hline Swan & 2010 & Prospective & $\begin{array}{l}\text { Chronic } \\
\text { radiation } \\
\text { proctitis }\end{array}$ & 49 & 1 & 98.0 & na & na \\
\hline Takemoto & 2012 & Prospective & $\begin{array}{l}\text { Hemorrhagic } \\
\text { radiation } \\
\text { proctopathy }\end{array}$ & 12 & 2 & 83.3 & na & na \\
\hline Tam & 2000 & Retrospective & Proctitis & 15 & 0 & 100.0 & $10.8 \pm 2.57$ & $13.3 \pm 1.84$ \\
\hline Tjandra & 2001 & Prospective & $\begin{array}{l}\text { Hemorrhagic } \\
\text { proctitis }\end{array}$ & 12 & 6 & 50.0 & $11.18 \pm 0.82$ & $12.28 \pm 0.55$ \\
\hline Venkatesh & 2002 & Prospective & $\begin{array}{c}\text { Radiation } \\
\text { proctitis }\end{array}$ & 40 & 1 & 97.5 & na & na \\
\hline Villavicencio & 2002 & Prospective & $\begin{array}{l}\text { Hemorrhagic } \\
\text { radiation } \\
\text { proctopathy }\end{array}$ & 21 & 0 & 100.0 & na & na \\
\hline Yeoh & 2013 & Prospective & $\begin{array}{l}\text { Chronic } \\
\text { radiation } \\
\text { proctitis }\end{array}$ & 17 & 1 & 94.1 & $14(97-159)$ & $13.6(10.6-17.4)$ \\
\hline Zinicola & 2003 & Retrospective & $\begin{array}{c}\text { Radiation } \\
\text { proctitis }\end{array}$ & 12 & 2 & 83.3 & na & na \\
\hline
\end{tabular}

and different amounts: from little sporadic spotting which leads, sometimes to chronic anemia state, to episodes of severe rectal bleeding [26].

APC is a nontouch electrocoagulation technique in which high-frequency alternating current can be delivered to the target lesion by ionized gas. The limited depth of coagulation $(0.5-3 \mathrm{~mm})$ [27] explained the low risk of perforation, stenosis, and fistulization. Unlike traditional bipolar devices, APC could be applied axially and radially, allowing tangential coagulation of lesions around rectal bends without significant reduction in effectiveness. Moreover, the APC generator is mobile and can be used quickly at any place or time [28]. Thus, APC is a well-established treatment for various conditions, such as oozing hemorrhage from angiodysplastic lesions or polypectomy sites.

Our study firstly confirmed that APC was an effective and safe therapy in endoscopically treating hemorrhagic CRP. APC therapy had a high success rate of hemostasis and low incidence of complications, which could help improve the hemoglobin levels. Furthermore, APC therapy could be performed at the outpatient clinics because no sedation or analgesia was required during the procedure [29]. Sedation with midazolam, fentanyl, or propofol was administrated to minimize the discomfort caused by the lesions near dentate line or gaseous distension of the rectum $[7,9,30]$.

Several studies reported that all patients had a decrease in transfusional requirements and an improvement in anemia $[6,7,10,16,25]$. The median lowest hemoglobin levels were $9.6 \mathrm{~g} / \mathrm{dL}$ (range 5.1-14.1) before APC and the median improvement in hemoglobin levels after treatment was $2.05 \mathrm{~g} / \mathrm{dL}$ (range $0.5-5.1$ ) in a study by Hortelano et al. [14]. As for safety, more than ten studies reported no complication during the follow-up $[5,15-17,25,31-36]$. However, several studies reported a low incidence of complications, including rectal or anal pain, recurrence of bleeding, rectal ulceration, and anal or rectal strictures. Rectal pain usually occurred near the dentate line after APC treatment [9]. It could be resolved spontaneously within a few days or with standard analgesics $[9,10,29]$. Recurrence of rectal bleeding was most likely to happen when patients were on anticoagulants or aspirin, and it also could be successfully treated after additional APC treatment. Although rectal ulcer and stenosis were common long-term complications, they were generally asymptomatic and did not require any additional endoscopic follow-up. In this systematic review, necrosis of the lower part of the rectum was reported in only one study [10]. 2 studies $[10,18]$ reported perforation following APC with low incidence. Ben et al. reported that one patient sustained a colonic explosion complicated by perforation that needed urgent surgery. The pathophysiology of the colon explosion remained unclear, but an accumulation of colonic gas (hydrogen and methane) at potentially explosive concentrations [37-39] could be the cause, especially the presence of stools above the lesions.

The optimal number of treatment sessions was still unknown. APC was traditionally not applied in one treatment session, particularly in patients with severe diseases. For therapeutic success, the median number of sessions per patient ranged from 1 to 3.7. Swan et al. [23] documented that there was a significant improvement in rectal bleeding among $68 \%$ of patients after the first session and $96 \%$ after two sessions. Karamanolis et al. [40] reported that APC with 2 sessions could resolve rectal bleeding completely in $89.3 \%$ (50/56) patients. According to this systematic review, the mean value of APC sessions was 1.9. 
TABLE 3: Study characteristics of selected studies on short-term and long-term complications after APC treatment.

\begin{tabular}{|c|c|c|c|c|c|c|}
\hline Author & Proctitis & $\begin{array}{l}\text { Total } \\
\text { number } \\
\text { of } \\
\text { bleeding }\end{array}$ & Short-term complications & $\begin{array}{l}\text { Number of } \\
\text { perforations }\end{array}$ & Long-term complications & $\begin{array}{c}\text { Rate of } \\
\text { complications } \\
(\%)\end{array}$ \\
\hline Alfadhli & Prospective & 14 & $\begin{array}{l}2 \text { (vomiting, abdominal cramps, } \\
\text { rectal pain, and fever) }\end{array}$ & 0 & 0 & 14.30 \\
\hline Álvaro-Villegas & $\begin{array}{l}\text { Chronic } \\
\text { radiation } \\
\text { proctitis }\end{array}$ & 14 & 2 (rectal pain) & 0 & 3 (rectal ulcers) & 35.70 \\
\hline Ben & $\begin{array}{l}\text { Hemorrhagic } \\
\text { radiation } \\
\text { proctitis }\end{array}$ & 27 & $\begin{array}{l}5 \text { (anal or rectal pain, vagal } \\
\text { symptoms, and colonic } \\
\text { explosions without perforation } \\
\text { in } 2 \text { and perforation in } 1 \text { ) }\end{array}$ & 1 & 0 & 18.50 \\
\hline Canard & $\begin{array}{l}\text { Radiation } \\
\text { proctitis }\end{array}$ & 30 & 6 (post treatment pain) & 1 & $\begin{array}{l}1 \text { (extensive necrosis of } \\
\text { the lower part of the } \\
\text { rectum) }\end{array}$ & 26.70 \\
\hline $\begin{array}{l}\text { Chruscielewska- } \\
\text { Kiliszek }\end{array}$ & $\begin{array}{l}\text { Chronic } \\
\text { radiation } \\
\text { proctitis }\end{array}$ & 62 & 1 (adynamic ileus) & 0 & $\begin{array}{l}35 \text { (asymptomatic rectal } \\
\text { ulcer in } 30 \text { and } \\
\text { symptomatic rectal ulcers } \\
\text { in 5) }\end{array}$ & 58.10 \\
\hline Chutkan & Proctitis & 12 & 0 & 0 & 0 & 0.00 \\
\hline $\begin{array}{l}\text { de la Serna } \\
\text { Higuera }\end{array}$ & $\begin{array}{l}\text { Hemorrhagic } \\
\text { radiation } \\
\text { proctopathy }\end{array}$ & 10 & 0 & 0 & 0 & 0.00 \\
\hline Dees & $\begin{array}{l}\text { Chronic } \\
\text { radiation } \\
\text { proctitis }\end{array}$ & 48 & 0 & 0 & $\begin{array}{l}1 \text { (recurrence of rectal } \\
\text { bleeding) }\end{array}$ & 2.00 \\
\hline Fantin & Proctitis & 7 & 0 & 0 & 0 & 0.00 \\
\hline Hortelano E & $\begin{array}{l}\text { Chronic } \\
\text { radiation } \\
\text { proctitis }\end{array}$ & 30 & 1 (incontinence) & 0 & 1 (rectal ulcer) & 6.67 \\
\hline Kaassis & Proctitis & 16 & $\begin{array}{c}4 \text { (transitory and minimal } \\
\text { dysenteric) }\end{array}$ & 0 & $\begin{array}{c}1 \text { (recurrence of rectal } \\
\text { bleeding) }\end{array}$ & 31.30 \\
\hline Karamanolis & $\begin{array}{l}\text { Radiation } \\
\text { proctitis }\end{array}$ & 56 & $\begin{array}{l}1 \text { (colonic explosion without } \\
\text { perforation) }\end{array}$ & 0 & $\begin{array}{c}2 \text { (recurrence of rectal } \\
\text { bleeding) }\end{array}$ & 5.4 \\
\hline Latorre & $\begin{array}{l}\text { Chronic } \\
\text { radiation } \\
\text { proctopathy }\end{array}$ & 38 & na & na & 0 & 0.00 \\
\hline Lenz & $\begin{array}{l}\text { Chronic } \\
\text { radiation } \\
\text { coloproctopathy }\end{array}$ & 15 & $\begin{array}{l}4 \text { (anal pain in } 2 \text { cases and } \\
\text { abdominal discomfort in } 1, \\
\text { worsening of bleeding during } \\
\text { treatment in } 1 \text { ) }\end{array}$ & 0 & $\begin{array}{l}2 \text { (tapered feces without } \\
\text { stenosis in } 1, \\
\text { asymptomatic stenosis in } \\
1 \text { ) }\end{array}$ & 40.00 \\
\hline Lpoez & $\begin{array}{l}\text { Radiation } \\
\text { proctopathy }\end{array}$ & 19 & 0 & 0 & $\begin{array}{l}1 \text { (recurrence of rectal } \\
\text { bleeding) }\end{array}$ & 5.30 \\
\hline Onoyama & $\begin{array}{l}\text { Chronic } \\
\text { hemorrhagic } \\
\text { radiation } \\
\text { proctitis }\end{array}$ & 24 & 0 & 0 & 0 & 0.00 \\
\hline Rolachon A & $\begin{array}{c}\text { Proctitis and } \\
\text { proctosigmoiditis }\end{array}$ & 12 & 0 & 0 & $\begin{array}{l}3 \text { (chronic rectal } \\
\text { ulcerations in } 2 \text { cases and } \\
\text { rectal stenosis in } 1 \text { case) }\end{array}$ & 25.00 \\
\hline Rotondano & $\begin{array}{l}\text { Chronic } \\
\text { radiation } \\
\text { proctopathy }\end{array}$ & 24 & $\begin{array}{l}5 \text { (mild bloating, cramping, anal } \\
\text { pain) }\end{array}$ & 0 & 1 (rectal stenosis) & 25.00 \\
\hline Sait Dag & $\begin{array}{l}\text { Radiation } \\
\text { proctitis }\end{array}$ & 21 & 4 (rectal pain and distension) & 0 & $\begin{array}{l}1 \text { (recurrence of rectal } \\
\text { bleeding) }\end{array}$ & 23.80 \\
\hline Samy & Chronic proctitis & 23 & na & na & 0 & 0.00 \\
\hline
\end{tabular}


TABle 3: Continued.

\begin{tabular}{|c|c|c|c|c|c|c|}
\hline Author & Proctitis & $\begin{array}{c}\text { Total } \\
\text { number } \\
\text { of } \\
\text { bleeding }\end{array}$ & Short-term complications & $\begin{array}{l}\text { Number of } \\
\text { perforations }\end{array}$ & Long-term complications & $\begin{array}{c}\text { Rate of } \\
\text { complications } \\
(\%)\end{array}$ \\
\hline Sarah & Proctitis & 11 & 0 & 0 & $\begin{array}{c}7 \text { (rectal stenosis in } 2 \\
\text { patients, ulceration in } 1, \\
\text { asymptomatic superficial } \\
\text { ulceration in } 4)\end{array}$ & 63.60 \\
\hline Sato & $\begin{array}{l}\text { Hemorrhagic } \\
\text { radiation } \\
\text { proctopathy }\end{array}$ & 65 & 8 (rectal pain) & 0 & $\begin{array}{l}4 \text { (recurrence of rectal } \\
\text { bleeding) }\end{array}$ & 18.50 \\
\hline Sebastian & $\begin{array}{l}\text { Radiation } \\
\text { proctopathy }\end{array}$ & 25 & 0 & 0 & 0 & 0.00 \\
\hline Silva & Proctosigmoiditis & 28 & 3 (transient anal pain) & 0 & 0 & 10.70 \\
\hline Smith & Proctitis & 7 & 0 & 0 & 0 & 0.00 \\
\hline Swan & $\begin{array}{l}\text { Chronic } \\
\text { radiation } \\
\text { proctitis }\end{array}$ & 49 & $\begin{array}{l}17 \text { (proctalgia in } 13 \text { patients, } \\
\text { rectal mucous discharge in } 4 \text {, } \\
\text { incontinence in } 1, \text { fever in } 1 \text {, and } \\
\text { bleeding in } 1 \text { ) }\end{array}$ & 0 & 1 (rectal stricture) & 36.00 \\
\hline Takemoto & $\begin{array}{l}\text { Hemorrhagic } \\
\text { radiation } \\
\text { proctopathy }\end{array}$ & 12 & 0 & 0 & 0 & 0.00 \\
\hline Tam & Proctitis & 15 & 0 & 0 & 2 (rectal stricture) & 13.30 \\
\hline Tjandra & $\begin{array}{c}\text { Hemorrhagic } \\
\text { proctitis }\end{array}$ & 12 & 0 & 0 & 0 & 0.00 \\
\hline Venkatesh & $\begin{array}{l}\text { Radiation } \\
\text { proctitis }\end{array}$ & 40 & 0 & 0 & 0 & 0.00 \\
\hline Villavicencio & $\begin{array}{l}\text { Hemorrhagic } \\
\text { radiation } \\
\text { proctopathy }\end{array}$ & 21 & $\begin{array}{l}3 \text { (rectal pain, tenesmus, and/or } \\
\text { abdominal distention) }\end{array}$ & 0 & $\begin{array}{l}1 \text { (recurrence of rectal } \\
\text { bleeding) }\end{array}$ & 19.00 \\
\hline Zinicola & $\begin{array}{c}\text { Radiation } \\
\text { proctitis }\end{array}$ & 12 & 0 & 0 & 1 (rectosigmoid stenosis) & 7.10 \\
\hline
\end{tabular}

As for APC setting, there was still no consensus for the optimal APC settings (power and gas flow rate) for successful and safe coagulation. Sato et al. [11] reported that the ideal coagulation time was 2 seconds from an ex vivo experiment on swine rectal mucosa. Weiner et al. [41] also found the impact of coagulation time on the depth and diameter of the coagulation zone and reported that as coagulation time and electric power increase, at larger electric power $(>75 \mathrm{~W})$ and/or longer coagulation time ( $>3 \mathrm{~s}$ ), the formation of craters and artificial clefts in submucosa and perforation increase. Because the data of coagulation time were not available in included studies, we could not conduct an analysis which could explain the effect of coagulation time on the depth. To sum up, coagulation time ranged from $0.5 \mathrm{~s}$ to $3 \mathrm{~s}$ in our study. Canard et al. reported that lower power settings were subscribed for a lower complication rate and decreased number of treatment sessions required for complete coagulation, with almost all complications occurring at power settings above $45 \mathrm{~W}$ [10]. We generally thought that lower power settings caused less injury, while in this systematic review, APC settings seemed to have no impact on complications.

We intended to further study the difference between the ICC system (e.g., first-generation APC) and the second- generation APC. Unfortunately, all of the trials included used the ICC system with parameter settings that were distinctly different from the second-generation APC. Compared with the ICC system, the second-generation APC offered a broader bandwidth of parameters including different APC modes and a range of power settings from 1 to $120 \mathrm{~W}$ [42]. Different APC modes appeared to be safe and effective in a variety of gastrointestinal conditions $[43,44]$.

Other than APC, salicylates, corticosteroids, sucralfate, and short-chain fatty acid enemas have been used with limited success, but hyperbaric oxygen therapy (HOT) and formalin application were shown to be effective in radiation proctitis $[4,5,45-48]$.

The mechanism of HOT in treating radiation tissue injury was the induction of neovascularization which could reverse tissue hypoxia. Macrophages responding to the oxygen gradient between the damaged hypoxic cells and the surrounding normal tissue mediated the stimulus for angiogenesis [49]. Tahir et al. [50] reported a 95\% efficacy of HOT for hemorrhagic CRP, where around half of the cases had a durable major response. Some patients even experienced symptom relief lasting as long as seven years. However, before consenting to HOT, patients should consider these factors: (1) Pressure inside the hyperbaric chamber can 
TABle 4: Newcastle-Ottawa Scale for assessing quality of cohort studies.

\begin{tabular}{|c|c|c|c|c|c|c|c|}
\hline Quality assessment scale & 1 & 2 & 3 & 4 & 5 & 6 & Total $(\max =6)$ \\
\hline Ben (2004) & $*$ & $*$ & $*$ & $*$ & $*$ & $*$ & 6 \\
\hline Canard (2003) & $*$ & $*$ & $*$ & $*$ & $*$ & $*$ & 6 \\
\hline Chruscielewska (2013) & $*$ & $*$ & $*$ & $*$ & $*$ & $*$ & 6 \\
\hline Chutkan (1997) & $*$ & $*$ & $*$ & $*$ & $*$ & $*$ & 6 \\
\hline Higuera (2006) & $*$ & $*$ & $*$ & - & $*$ & $*$ & 5 \\
\hline Dees (1999) & $*$ & $*$ & $*$ & $*$ & $*$ & - & 5 \\
\hline Fantin (2004) & $*$ & $*$ & $*$ & $*$ & $*$ & $*$ & 6 \\
\hline Hortelano (2014) & $*$ & $*$ & $*$ & $*$ & $*$ & $*$ & 6 \\
\hline Kaassis (2002) & $*$ & $*$ & $*$ & $*$ & $*$ & $*$ & 6 \\
\hline Karamanolis (2009) & $*$ & $*$ & $*$ & $*$ & $*$ & $*$ & 6 \\
\hline Latorre (2008) & $*$ & $*$ & $*$ & $*$ & $*$ & $*$ & 6 \\
\hline Lpoez (2010) & $*$ & $*$ & $*$ & $*$ & $*$ & $*$ & 6 \\
\hline Onoyama (2011) & $*$ & $*$ & $*$ & $*$ & $*$ & $*$ & 6 \\
\hline Rolachon (2000) & $*$ & $*$ & $*$ & $*$ & $*$ & $*$ & 6 \\
\hline Rotondano (2003) & $*$ & $*$ & $*$ & $*$ & $*$ & $*$ & 6 \\
\hline Sait (2013) & $*$ & $*$ & $*$ & $*$ & $*$ & $*$ & 6 \\
\hline Samy (2012) & $*$ & $*$ & $*$ & - & - & $*$ & 4 \\
\hline Sarah (2001) & $*$ & $*$ & $*$ & $*$ & - & $*$ & 5 \\
\hline Sato (2011) & $*$ & $*$ & $*$ & $*$ & $*$ & $*$ & 6 \\
\hline Sebastian (2004) & $*$ & $*$ & $*$ & - & $*$ & $*$ & 5 \\
\hline Silva (1999) & $*$ & $*$ & $*$ & $*$ & $*$ & $*$ & 6 \\
\hline Smith (2001) & $*$ & $*$ & $*$ & $*$ & - & $*$ & 5 \\
\hline Swan (2010) & $*$ & $*$ & $*$ & $*$ & $*$ & $*$ & 6 \\
\hline Takemoto (2012) & $*$ & $*$ & $*$ & $*$ & $*$ & $*$ & 6 \\
\hline Tam (2000) & $*$ & $*$ & $*$ & $*$ & $*$ & $*$ & 6 \\
\hline Tjandra (2001) & $*$ & $*$ & $*$ & $*$ & $*$ & $*$ & 6 \\
\hline Venkatesh (2002) & $*$ & $*$ & $*$ & - & - & $*$ & 4 \\
\hline Villavicencio (2002) & $*$ & $*$ & $*$ & $*$ & $*$ & $*$ & 6 \\
\hline Zinicola (2003) & $*$ & $*$ & $*$ & $*$ & $*$ & $*$ & 6 \\
\hline
\end{tabular}

1: representativeness of the exposed cohort; 2: ascertainment of APC exposure; 3: demonstration that outcome of interest was not present at start of study; 4: assessment of outcome; 5: was follow-up long enough for outcomes to occur; 6: adequacy of follow-up of cohorts. Asterisk (*) indicated one score and hyphen (-) indicated zero score.

damage the middle and inner ear, nasal sinuses, lungs, and teeth in both adults and children. (2) Some people experienced claustrophobia inside the chamber. (3) The therapy might affect your eyes, for example, by promoting nearsightedness or cataract growth. (4) Diabetics should have their levels checked before and after treatment, because hyperbaric oxygen therapy affects blood sugar levels. (5) The cost of HOT was high enough, and it was not widely applicable. There was no uniformity in the methods of HOT. Although it could be perceived from the studies that HOT was useful in refractory radiation proctitis, there was marked variation between the studies $[48,50-53]$. The reported number of
HOT sessions for a successful treatment ranges from 12 to 90 . Recently, a double-blind, sham-controlled, phase-3 randomized trial conducted by Glover et al. [54] reported that there was no significant difference between APC and HOT in rectal bleeding. Álvaro-Villegas et al. reported that APC and HOT were similar in treating rectal bleeding, while response rate was higher and faster in the APC group [13].

Formalin was a mixture of methanol and formaldehyde which covalently binded to proteins and causes cell necrosis. It acted as a hemostatic agent causing chemical cauterization to control bleeding from telangiectatic mucosal and submucosal vessels. In 1986, Rubinstein et al. were the first to use formalin for a hemorrhagic CRP patient to get a good response [55]. Most used 4\% dilute formalin applied to the rectum mucosa either by direct application of formalinsoaked gauze or by "instilling" the solution in single or multiple aliquots down the operating channel of a colonoscope. Guo et al. in their randomized trial which randomly divided 122 patients into $4 \%$ or $10 \%$ formalin application showed that $10 \%$ formalin was associated with complications and $4 \%$ formalin should be the choice for treating hemorrhagic CRP [56]. Alfadhli et al. [4] compared the efficacy of formalin instillation therapy with APC therapy and found that APC showed a better efficacy (78.5\% versus 27\%). Nevertheless, the study of Yeoh et al. [57] found that there was no statistical difference between the APC group and the formalin group (94\% versus $100 \%$, respectively). It was worth noting that in formalin therapy, $18 \%$ of the patients underwent intestinal stricture and $21 \%$ with fecal incontinence [58]. Therefore, formalin might be proper for patients with proctitis and refractoriness to other endoscopy therapies, like argon plasma coagulation, rather than an upfront approach.

Any randomized control trials on APC in treating hemorrhagic CRP could not be available, which had the underlying methodological limitations in this systematic review. In addition, individual patient data (IPD) from eligible trials on APC were not available because it might not be possible to contact the original trial authors or authors might not be willing to share the raw data. Furthermore, we included several trials published early.

In conclusion, APC is a safe and effective method for treating hemorrhagic CRP. Further evidence from randomized controlled trials and comparative studies is required to confirm the role of APC and APC settings, and APC should be considered as a first-line therapy for hemorrhagic CRP.

\section{Conflicts of Interest}

The authors declared no conflicts of interest in this work.

\section{Authors' Contributions}

Yanan Peng and Jing Liu conceived and designed the experiments. Yanan Peng, Juerong Feng, Haizhou Wang, Shilin Fang, and Fan Wang analyzed the data. Juerong Feng, Ying Chang, Meng Zhang, and Qiu Zhao contributed reagents/ materials/analysis tools. Yanan Peng, Haizhou Wang, Meng Zhang, and Jing Liu wrote the paper. Yanan Peng, Shilin 
Fang, Xianyan Shi, and Jing Liu collected important background information and acquired the data. All authors approved the final version of this paper. Yanan Peng and Haizhou Wang contributed equally to this work.

\section{Acknowledgments}

This work was supported by funds from the National Natural Science Foundation of China (Jing Liu, Grant no. 81472735), Wuhan Science and Technology Bureau (Jing Liu), Wuhan City Health and Family Planning Commission, Wuhan University (Jing Liu), and National Natural Science Fund no. 81472033 .

\section{References}

[1] R. Kochhar, P. V. Sriram, S. C. Sharma, R. C. Goel, and F. Patel, "Natural history of late radiation proctosigmoiditis treated with topical sucralfate suspension," Digestive Diseases and Sciences, vol. 44, no. 5, pp. 973-978, 1999.

[2] N. Y. Haboubi, P. F. Schofield, and P. L. Rowland, “The light and electron microscopic features of early and late phase radiation-induced proctitis," The American Journal of Gastroenterology, vol. 83, no. 10, pp. 1140-1144, 1988.

[3] S. A. Wilson and D. K. Rex, "Endoscopic treatment of chronic radiation proctopathy," Current Opinion in Gastroenterology, vol. 22, no. 5, pp. 536-540, 2006.

[4] A. A. Alfadhli, W. M. Alazmi, T. Ponich et al., "Efficacy of argon plasma coagulation compared to topical formalin application for chronic radiation proctopathy," Canadian Journal of Gastroenterology, vol. 22, no. 2, pp. 129-132, 2008.

[5] A. C. Fantin, J. Binek, W. R. Suter, and C. Meyenberger, "Argon beam coagulation for treatment of symptomatic radiation-induced proctitis," Gastrointestinal Endoscopy, vol. 49, no. 4, Part 1, pp. 515-518, 1999.

[6] M. Kaassis, E. Oberti, P. Burtin, and J. Boyer, “Argon plasma coagulation for the treatment of hemorrhagic radiation proctitis," Endoscopy, vol. 32, no. 9, pp. 673-676, 2000.

[7] W. Tam, J. Moore, and M. Schoeman, "Treatment of radiation proctitis with argon plasma coagulation," Endoscopy, vol. 32, no. 9, pp. 667-672, 2000.

[8] S. Taieb, A. Rolachon, J. C. Cenni et al., "Effective use of argon plasma coagulation in the treatment of severe radiation proctitis," Diseases of the Colon and Rectum, vol. 44, no. 12, pp. 1766-1771, 2001.

[9] R. T. Villavicencio, D. K. Rex, and E. Rahmani, "Efficacy and complications of argon plasma coagulation for hematochezia related to radiation proctopathy," Gastrointestinal Endoscopy, vol. 55, no. 1, pp. 70-74, 2002.

[10] J. M. Canard, B. Vedrenne, G. Bors, P. Claude, R. Bader, and D. Sondag, "Long term results of treatment of hemorrhagic radiation proctitis by argon plasma coagulation," Gastroentérologie Clinique et Biologique, vol. 27, no. 5, pp. 455-459, 2003.

[11] Y. Sato, T. Takayama, T. Sagawa et al., "Argon plasma coagulation treatment of hemorrhagic radiation proctopathy: the optimal settings for application and long-term outcome," Gastrointestinal Endoscopy, vol. 73, no. 3, pp. 543-549, 2011.
[12] J. J. Vargo, "Clinical applications of the argon plasma coagulator," Gastrointestinal Endoscopy, vol. 59, no. 1, pp. 81-88, 2004.

[13] J. C. Álvaro-Villegas, S. Sobrino-Cossio, L. C. Tenorio-Téllez et al., "Argon plasma coagulation and hyperbaric oxygen therapy in chronic radiation proctopathy, effectiveness and impact on tissue toxicity," Revista Española de Enfermedades Digestivas, vol. 103, no. 11, pp. 576-581, 2011, 22149559.

[14] E. Hortelano, A. Gomez-Iturriaga, R. Ortiz-de-Zarate et al., "Is argon plasma coagulation an effective and safe treatment option for patients with chronic radiation proctitis after high doses of radiotherapy?," Revista Española de Enfermedades Digestivas, vol. 106, no. 3, pp. 165-170, 2014.

[15] S. M. Al Gizawy, L. Abdelbaki, and N. A. Makhlouf, "Radiation induced-late bowel toxicity: role of argon plasma coagulation," Journal of Radiotherapy in Practice, vol. 11, no. 2, pp. 92-100, 2011.

[16] M. Onoyama, S. Nagata, T. Tsumura et al., "Effectiveness of argon plasma coagulation (APC) for hemorrhagic radiation proctitis," Digestive Endoscopy, vol. 23, no. 3, article A13, 2011.

[17] K. S. Venkatesh and P. Ramanujam, "Endoscopic therapy for radiation proctitis-induced hemorrhage in patients with prostatic carcinoma using argon plasma coagulator application," Surgical Endoscopy, vol. 16, no. 4, pp. 707-710, 2002.

[18] E. Ben-Soussan, M. Antonietti, G. Savoye, S. Herve, P. Ducrotté, and E. Lerebours, "Argon plasma coagulation in the treatment of hemorrhagic radiation proctitis is efficient but requires a perfect colonic cleansing to be safe," European Journal of Gastroenterology \& Hepatology, vol. 16, no. 12, pp. 1315-1318, 2004.

[19] K. E. Grund, C. Zindel, and G. Farin, “Argon plasma coagulation through a flexible endoscope: evaluation of a new therapeutic method after 1606 applications," Deutsche Medizinische Wochenschrift, vol. 122, no. 14, pp. 432-438, 1997.

[20] M. R. Chruscielewska-Kiliszek, J. Regula, M. Polkowski et al., "Sucralfate or placebo following argon plasma coagulation for chronic radiation proctitis: a randomized double blind trial," Colorectal Disease, vol. 15, no. 1, pp. e48-e55, 2013.

[21] G. M. Eisen, J. A. Dominitz, D. O. Faigel et al., "Endoscopic therapy of anorectal disorders," Gastrointestinal Endoscopy, vol. 53, no. 7, pp. 867-870, 2001.

[22] D. Ravizza, G. Fiori, C. Trovato, and C. Crosta, "Frequency and outcomes of rectal ulcers during argon plasma coagulation for chronic radiation-induced proctopathy," Gastrointestinal Endoscopy, vol. 57, no. 4, pp. 519-525, 2003.

[23] M. P. Swan, G. T. C. Moore, W. Sievert, and D. A. Devonshire, "Efficacy and safety of single-session argon plasma coagulation in the management of chronic radiation proctitis," Gastrointestinal Endoscopy, vol. 72, no. 1, pp. 150-154, 2010.

[24] J. Dees, M. A. C. Meijssen, and E. J. Kuipers, “Argon plasma coagulation for radiation proctitis," Scandinavian Journal of Gastroenterology, vol. 41, Supplement 243, pp. 175-178, 2006.

[25] C. de la Serna Higuera, M. Martin Arribas, S. Rodriguez Gomez, A. Perez Villoria, J. Martinez Moreno, and A. Betancourt Gonzalez, "Efficacy and safety of argon plasma coagulation for the treatment of hemorrhagic radiation proctitis," Revista Española de Enfermedades Digestivas, vol. 96, no. 11, pp. 758-764, 2004.

[26] F. C. A. den Hartog Jager, M. van Haastert, J. J. Batterman, and G. N. J. Tytgat, "The endoscopic spectrum of late radiation 
damage of the rectosigmoid colon," Endoscopy, vol. 17, no. 6, pp. 214-216, 1985.

[27] G. Farin and K. E. Grund, "Technology of argon plasma coagulation with particular regard to endoscopic applications," Endoscopic Surgery and Allied Technologies, vol. 2, no. 1, pp. 71-77, 1994.

[28] K. E. Grund, D. Storek, and G. Farin, "Endoscopic argon plasma coagulation (APC) first clinical experiences in flexible endoscopy," Endoscopic Surgery and Allied Technologies, vol. 2, no. 1, pp. 42-46, 1994.

[29] R. A. Silva, A. J. Correia, L. M. Dias, H. L. Viana, and R. L. Viana, "Argon plasma coagulation therapy for hemorrhagic radiation proctosigmoiditis," Gastrointestinal Endoscopy, vol. 50, no. 2, pp. 221-224, 1999.

[30] R. Zinicola, M. D. Rutter, G. Falasco et al., "Haemorrhagic radiation proctitis: endoscopic severity may be useful to guide therapy," International Journal of Colorectal Disease, vol. 18, no. 5, pp. 439-444, 2003.

[31] R. Chutkan, A. Lipp, and J. Waye, “The argon plasma coagulator: a new and effective modality for treatment of radiation proctitis," Gastrointestinal Endoscopy, vol. 45, no. 4, article AB27, 1997.

[32] M. Latorre Sánchez, J. Sempere García-Argüelles, S. Barceló Cerdá et al., "Evaluation of the endoscopic response to argon plasma coagulation in patients with chronic radiation proctopathy," Revista Española de Enfermedades Digestivas, vol. 100, no. 10, pp. 619-624, 2008.

[33] S. Sebastian, H. O'Connor, C. O'Morain, and M. Buckley, "Argon plasma coagulation as first-line treatment for chronic radiation proctopathy," Journal of Gastroenterology and Hepatology, vol. 19, no. 10, pp. 1169-1173, 2004.

[34] S. Smith, K. Wallner, J. A. Dominitz et al., "Argon plasma coagulation for rectal bleeding after prostate brachytherapy," International Journal of Radiation Oncology, Biology, Physics, vol. 51, no. 3, pp. 636-642, 2001.

[35] J. J. Tjandra and S. Sengupta, “Argon plasma coagulation is an effective treatment for refractory hemorrhagic radiation proctitis," Diseases of the Colon \& Rectum, vol. 44, no. 12, pp. 17591765, 2001.

[36] S. Takemoto, Y. Shibamoto, S. Ayakawa et al., "Treatment and prognosis of patients with late rectal bleeding after intensitymodulated radiation therapy for prostate cancer," Radiation Oncology, vol. 7, no. 1, p. 87, 2012.

[37] A. Avgerinos, N. Kalantzis, G. Rekoumis, G. Pallikaris, G. Arapakis, and T. Kanaghinis, "Bowel preparation and the risk of explosion during colonoscopic polypectomy," Gut, vol. 25, no. 4, pp. 361-364, 1984.

[38] M. A. Bigard, P. Gaucher, and C. Lassalle, "Fatal colonic explosion during colonoscopic polypectomy," Gastroenterology, vol. 77, no. 6, pp. 1307-1310, 1979.

[39] S. J. La Brooy, A. Avgerinos, C. L. Fendick, C. B. Williams, and J. J. Misiewicz, "Potentially explosive colonic concentrations of hydrogen after bowel preparation with mannitol," The Lancet, vol. 1, no. 8221, pp. 634-636, 1981.

[40] G. Karamanolis, K. Triantafyllou, Z. Tsiamoulos et al., "Argon plasma coagulation has a long-lasting therapeutic effect in patients with chronic radiation proctitis," Endoscopy, vol. 41, no. 6, pp. 529-531, 2009.

[41] J. P. Weiner, A. T. Wong, D. Schwartz, M. Martinez, A. Aytaman, and D. Schreiber, "Endoscopic and nonendoscopic approaches for the management of radiation- induced rectal bleeding," World Journal of Gastroenterology, vol. 22, no. 31, pp. 6972-6986, 2016.

[42] H. Manner, A. May, M. Faerber, O. Pech, N. Plum, and C. Ell, "The tissue effect of second generation argon plasma coagulation (VIO APC) in comparison to standard APC and Nd:YAG laser in vitro," Acta Gastroenterologica Belgica, vol. 70, no. 4, pp. 352-356, 2007.

[43] H. Manner, "Argon plasma coagulation therapy," Current Opinion in Gastroenterology, vol. 24, no. 5, pp. 612-616, 2008.

[44] H. Manner, M. D. Enderle, O. Pech et al., "Second-generation argon plasma coagulation: two-center experience with 600 patients," Journal of Gastroenterology and Hepatology, vol. 23, no. 6, pp. 872-878, 2008.

[45] S. D. Ladas and S. A. Raptis, "Sucralfate enemas in the treatment of chronic postradiation proctitis," The American Journal of Gastroenterology, vol. 84, no. 12, pp. 1587-1589, 1989.

[46] R. Kochhar, F. Patel, A. Dhar et al., "Radiation-induced proctosigmoiditis: prospective, randomized, double-blind controlled trial of oral sulfasalazine plus rectal steroids versus rectal sucralfate," Digestive Diseases and Sciences, vol. 36, no. 1, pp. 103-107, 1991.

[47] N. A. Talley, F. Chen, D. King, M. Jones, and N. J. Talley, "Short-chain fatty acids in the treatment of radiation proctitis: a randomized, double-blind, placebo-controlled, cross-over pilot trial," Diseases of the Colon and Rectum, vol. 40, no. 9, pp. 1046-1050, 1997.

[48] S. Girnius, N. Cersonsky, L. Gesell, S. Cico, and W. Barrett, "Treatment of refractory radiation-induced hemorrhagic proctitis with hyperbaric oxygen therapy," American Journal of Clinical Oncology, vol. 29, no. 6, pp. 588-592, 2006.

[49] D. R. Knighton, T. K. Hunt, H. Scheuenstuhl, B. J. Halliday, Z. Werb, and M. J. Banda, "Oxygen tension regulates the expression of angiogenesis factor by macrophages," Science, vol. 221, no. 4617, pp. 1283-1285, 1983.

[50] A. R. M. Tahir, J. Westhuyzen, J. Dass et al., "Hyperbaric oxygen therapy for chronic radiation-induced tissue injuries: Australasia's largest study," Asia-Pacific Journal of Clinical Oncology, vol. 11, no. 1, pp. 68-77, 2015.

[51] D. C. Warren, P. Feehan, J. B. Slade, and P. E. Cianci, "Chronic radiation proctitis treated with hyperbaric oxygen," Undersea \& Hyperbaric Medicine, vol. 24, no. 3, pp. 181184, 1997.

[52] K. Jones, A. W. Evans, R. G. Bristow, and W. Levin, "Treatment of radiation proctitis with hyperbaric oxygen," Radiotherapy \& Oncology, vol. 78, no. 1, pp. 91-94, 2006.

[53] M. A. Dall'Era, N. B. Hampson, R. A. Hsi, B. Madsen, and J. M. Corman, "Hyperbaric oxygen therapy for radiation induced proctopathy in men treated for prostate cancer," The Journal of Urology, vol. 176, no. 1, pp. 87-90, 2006.

[54] M. Glover, G. R. Smerdon, H. J. Andreyev et al., "Hyperbaric oxygen for patients with chronic bowel dysfunction after pelvic radiotherapy (HOT2): a randomised, double-blind, sham-controlled phase 3 trial," The Lancet Oncology, vol. 17, no. 2, pp. 224-233, 2016.

[55] E. Rubinstein, T. Ibsen, R. B. Rasmussen, E. Reimer, and B. L. Sorensen, "Formalin treatment of radiation-induced hemorrhagic proctitis," American Journal of Gastroenterology, vol. 81, no. 1, pp. 44-45, 1986. 
[56] G. H. Guo, F. Y. Yu, X. J. Wang, and F. Lu, “A randomized controlled clinical trial of formalin for treatment of chronic hemorrhagic radiation proctopathy in cervical carcinoma patients," Supportive Care in Cancer, vol. 23, no. 2, pp. 441446, 2015.

[57] E. Yeoh, W. Tam, M. Schoeman et al., “Argon plasma coagulation therapy versus topical formalin for intractable rectal bleeding and anorectal dysfunction after radiation therapy for prostate carcinoma," International Journal of Radiation Oncology, Biology, Physics, vol. 87, no. 5, pp. 954-959, 2013.

[58] V. de Parades, I. Etienney, P. Bauer et al., "Formalin application in the treatment of chronic radiation-induced hemorrhagic proctitis-an effective but not risk-free procedure: a prospective study of 33 patients," Diseases of the Colon \& Rectum, vol. 48, no. 8, pp. 1535-1541, 2005. 


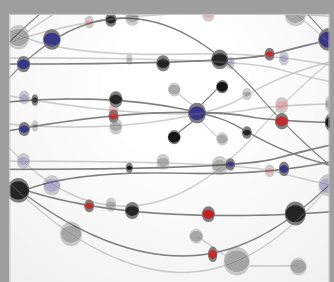

The Scientific World Journal
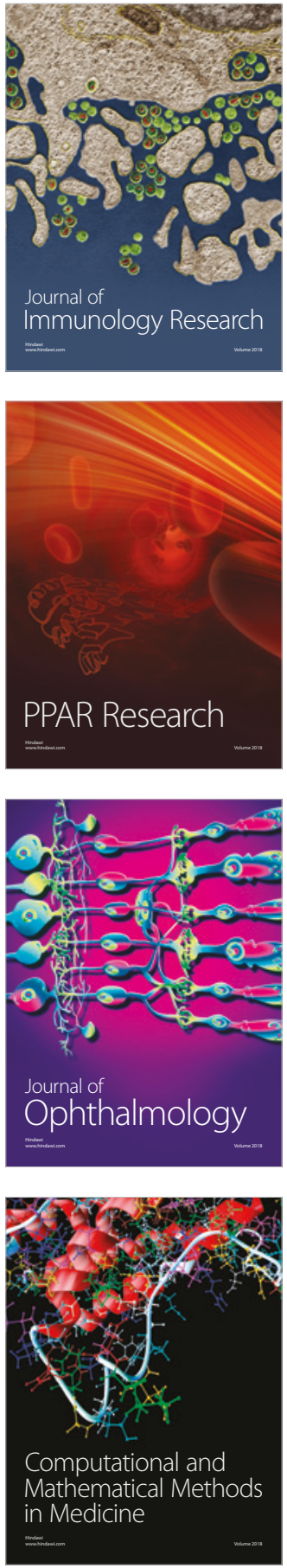

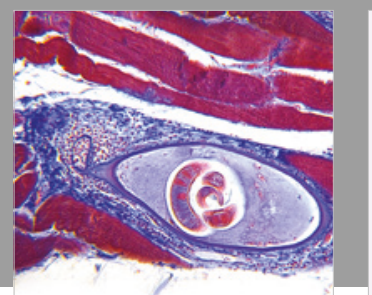

Gastroenterology Research and Practice

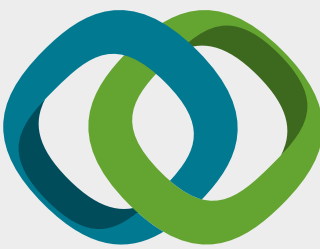

\section{Hindawi}

Submit your manuscripts at

www.hindawi.com
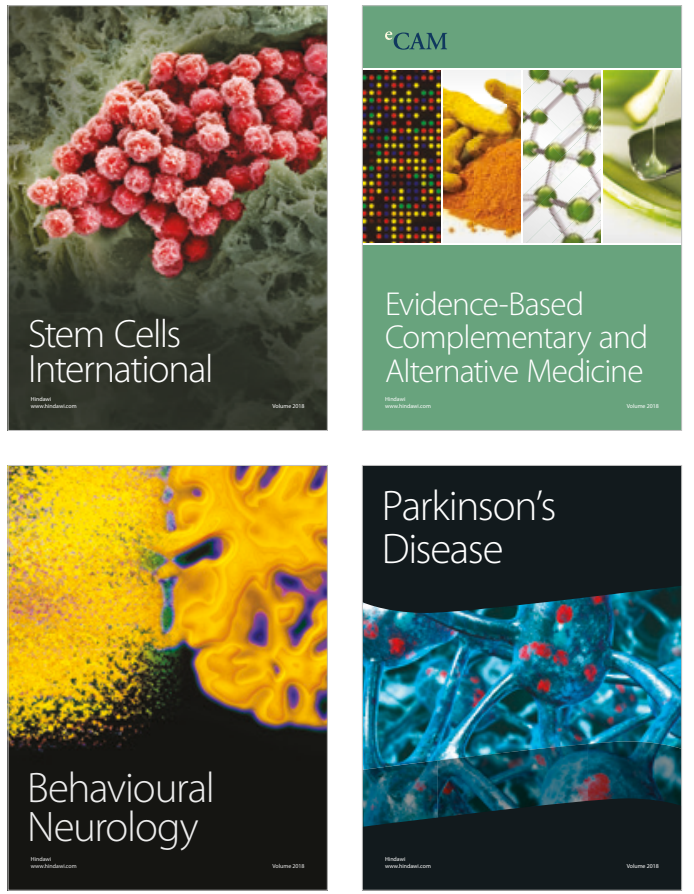

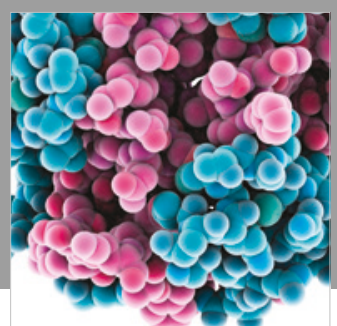

ournal of

Diabetes Research

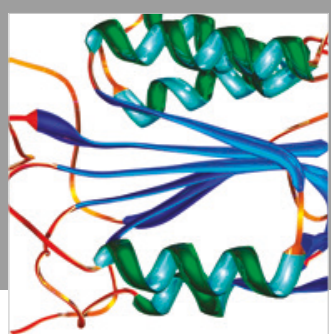

Disease Markers
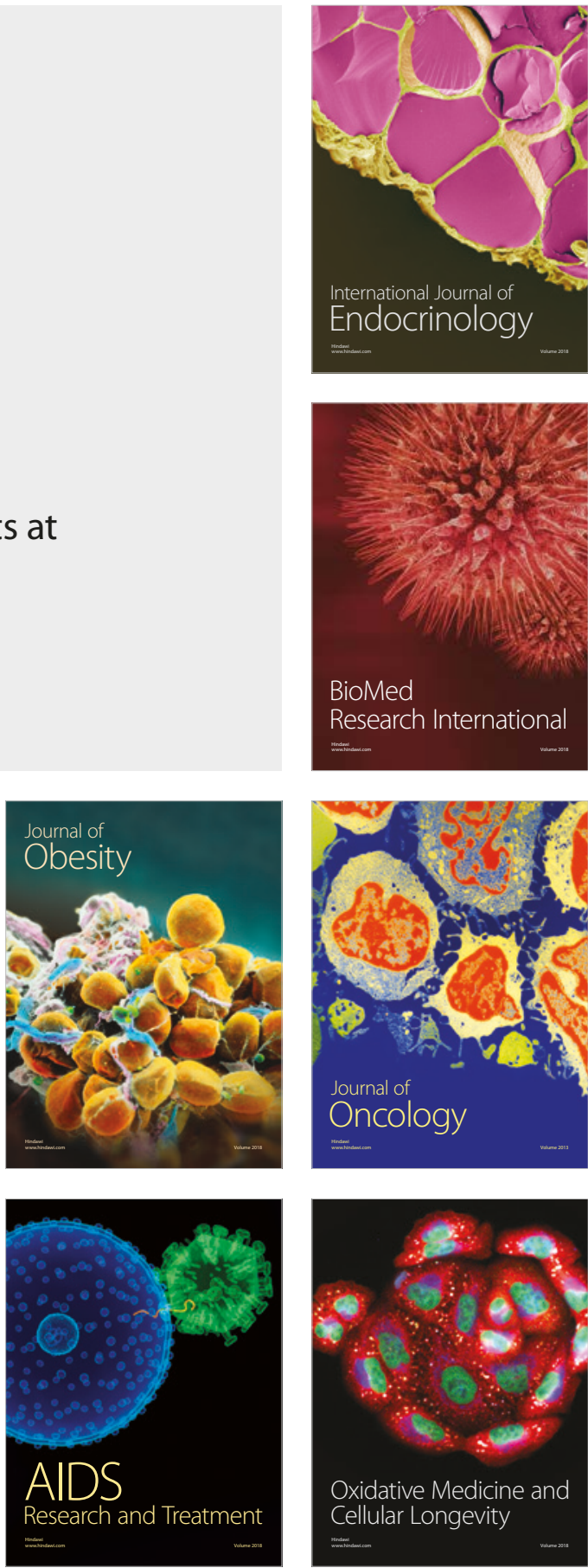